\title{
Neurobiologie und Stoffwechsel
}

Zunehmend erschließt sich uns derzeit die Notwendigkeit, den Stoffwechsel und den neurobiologischen Zustand eines menschlichen Organismus genauer ins Blickfeld zu rücken. Wie bei einem Auto, das nur mit ausreichend Benzin, gutem Öl, dem richtigen Kühlwasser und einem gefüllten Scheibenwassertank gut nutzbar ist, so verhält es sich auch mit unserem Körper. Fehlen ihm wichtige Substanzen, Vitamine oder Nahrungsbestandteile, wird er zunehmend schlechter fahren. Dies hat Auswirkungen auch auf die Psyche, die dann nicht mehr für ein ausgewogenes psychisches Wohlempfinden sorgen kann.

Dr. Roger Ziegler aus der Schweiz befasst sich bereits seit vielen Jahren mit der chinesischen und tibetischen Medizin und hat begonnen, sein Wissen auf die speziellen Bedürfnisse hochsensibler Menschen anzuwenden. So betont er, dass es wichtig ist, immer zuerst das Gehirn als die Schaltstelle neurobiologischer Prozesse zu stärken (,kühlen ' in der östliche Sprache) und damit lern- und bewegungsfähig, veränderungs- und therapiefähig zu machen. Das Gehirn wird dadurch wieder regulier- und steuerungsfähig und kann dann sehr schnell und präzise die physischen, emotionalen, mentalen und spirituellen Reize energiesparend sortieren.

Dies kann zum Beispiel durch die Aufnahme von Nahrungsergänzungsmittel und Vitamine erreicht werden.

Es gibt allerdings auch andere heilende Fachkräfte, die um die Notwendigkeit eines ausgewogenen Stoffwechsels wissen und Hilfe anbieten können. Fragen Sie dazu in Ihrem Umfeld nach entsprechenden Erfahrungen und Anlaufadressen. 